\title{
Clostridial Mycotic Aneurysm Leading to Emphysematous Aortitis
}

\author{
Thomas G. $\mathrm{Ng}^{1}$, Usha Trivedi ${ }^{1}$, Kajol Shah ${ }^{1}$, Pierre Maldjian ${ }^{2}$ \\ 1. Internal Medicine, Rutgers University, Newark, USA 2. Radiology, Rutgers University, Newark, USA
}

Corresponding author: Thomas G. Ng, tgn14@njms.rutgers.edu

\begin{abstract}
Mycotic aneurysms account for less than $5 \%$ of all aneurysms of the aorta, with most cases linked to infection with either Staphylococcus or Salmonella species. Emphysematous aortitis is a rare consequence of mycotic aneurysms and is associated with high morbidity and mortality. It typically occurs from infection superimposed on already damaged endothelium, which is commonly seen in conditions such as atherosclerosis. This report discusses the presentation and relevant imaging findings of a unique case of emphysematous aortitis from Clostridial infection of the thoracic aorta. The patient was a 66-year-old male with a past medical history of end-stage renal disease, arteriovenous fistula for dialysis, hypertension, and diabetes, who presented with tachycardia and tachypnea. Computed tomography of the chest showed inflammatory changes of the thoracic aorta with gas bubbles along the aortic wall, and post-mortem aortic tissue cultures were positive for Clostridium innocuum. Although emphysematous aortitis is rare, the radiographic findings are strikingly characteristic and should prompt immediate and aggressive management.
\end{abstract}

Categories: Cardiac/Thoracic/Vascular Surgery, Infectious Disease, Anatomy

Keywords: emphysematous aortitis, clostridium, mycotic aneurysm, rutgers njms, cardiothoracic surgery

\section{Introduction}

Emphysematous aortitis is a rare consequence of underlying mycotic aneurysm, an uncommon cause of arterial dilation resulting from infection of damaged vessel endothelium. A misnomer, mycotic aneurysm is usually caused by bacterial rather than fungal infection, and accounts for $0.7 \%-1.3 \%$ of all surgically treated aneurysms. Common risk factors include immunosuppression, intravenous drug use, iatrogenic vessel trauma, atherosclerosis, and endocarditis. This phenomenon can not only affect the thoracic and abdominal aorta but also the intracranial and peripheral arteries. Most cases have been linked to infection with either Staphylococcus or Salmonella species [1]. This case reports Clostridial aortic infection, an uncommon cause of mycotic aneurysm, leading to arterial myonecrosis and subsequent aortitis diagnosed on computed tomography (CT) imaging.

Review began 03/18/2021 Review ended 03/23/2021 Published 03/27/2021

() Copyright 2021 $\mathrm{Ng}$ et al. This is an open access article distributed under the terms of the Creative Commons Attribution License CC-BY 4.0., which permits unrestricted use, distribution, and reproduction in any medium, provided the original author and source are credited.

\section{Case Presentation}

The patient was a 66-year-old man with a past medical history of end-stage renal disease with an arteriovenous fistula placed five years prior in the left arm for dialysis, hypertension, and diabetes who presented with one-week history of non-bloody diarrhea, vomiting, and fever. On physical examination, the patient was tachycardic and tachypneic. The patient's initial laboratory results were significant for a leukocytosis of $23,000 / \mu \mathrm{L}$ with elevated inflammatory markers including a D-dimer of 4,700 ng/mL, a Creactive protein of $382 \mathrm{mg} / \mathrm{L}$, and a procalcitonin of $25.26 \mathrm{ng} / \mathrm{mL}$. An initial chest radiograph was significant for a widened mediastinum with a lucent region alongside the thoracic aorta (Figure 1). Computed tomography showed a $1.4 \mathrm{~cm}$ aneurysmal out-pouching of the proximal descending thoracic aorta along with inflammatory changes and gas bubbles in the periaortic soft tissues extending from the aortic arch to the mid descending thoracic aorta diagnostic of emphysematous aortitis (Figures 2-4). There was also a large, loculated, left-sided pleural effusion with atelectasis of the left lower lobe initially concerning for empyema, but likely reactive as thoracentesis revealed polymorphonuclear lymphocytes with negative bacterial cultures. 


\section{Cureus}

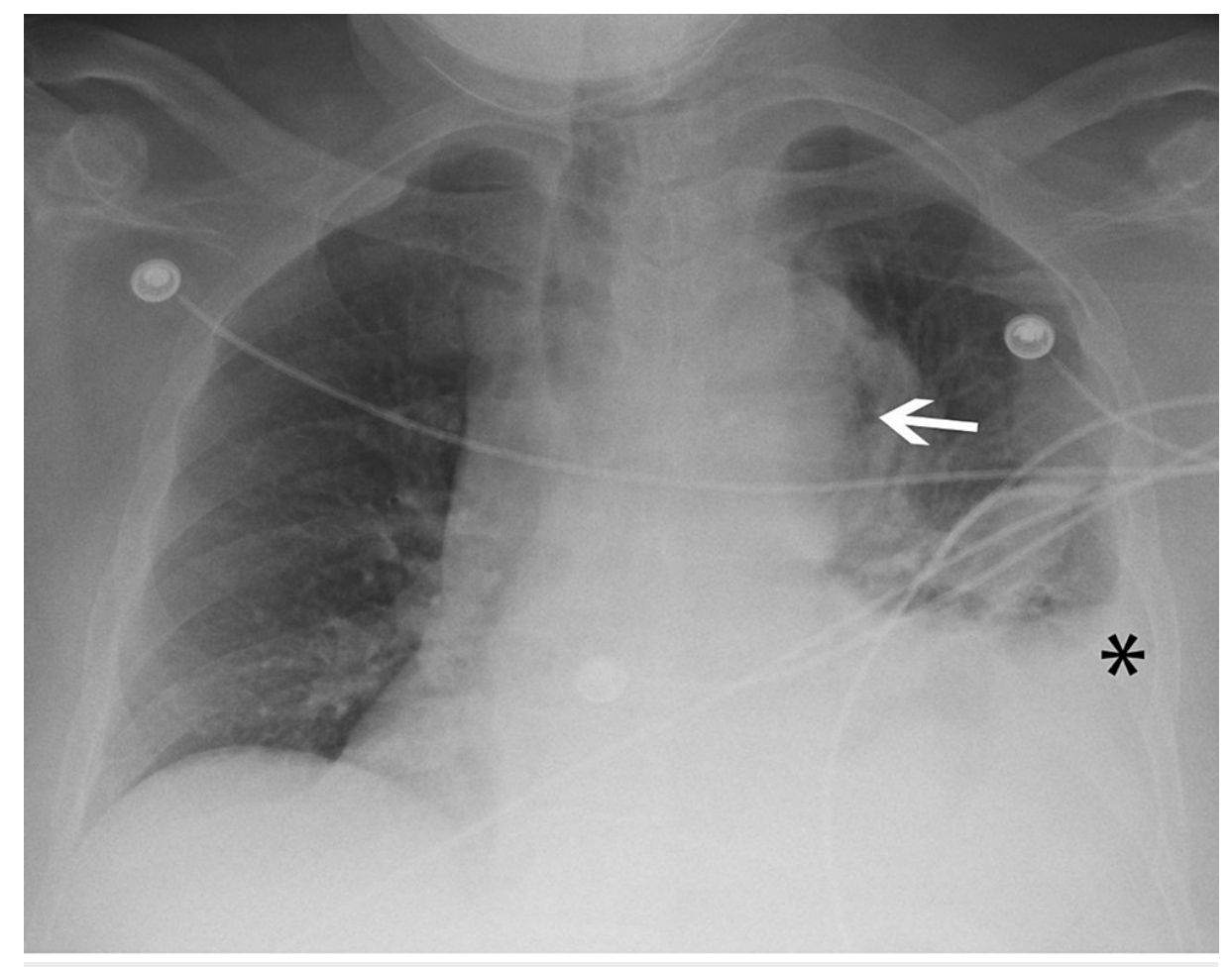

FIGURE 1: Chest radiograph shows widening of the mediastinum with a lucent region (arrow) along the left border of the thoracic aorta. Note left pleural effusion $(*)$.

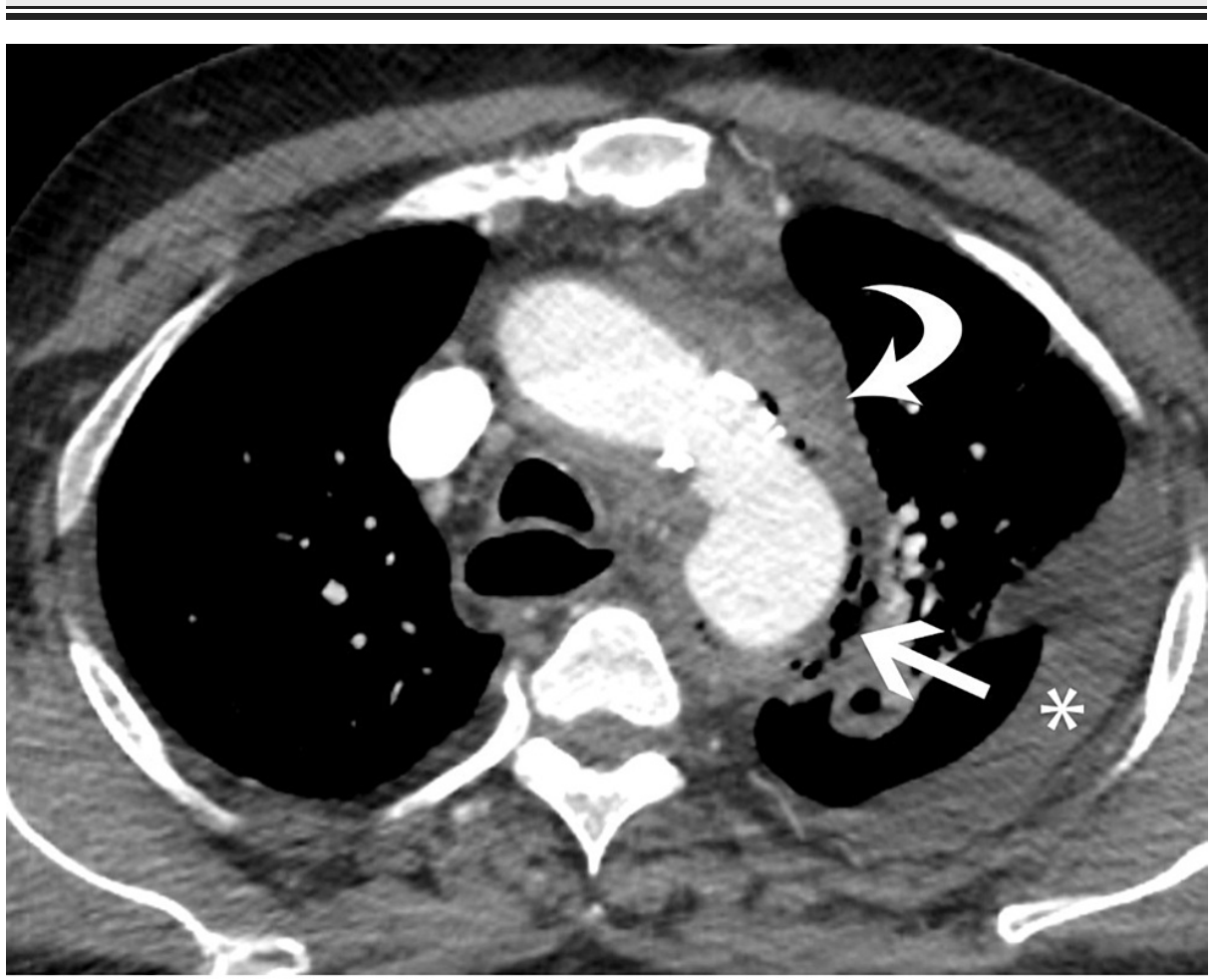

FIGURE 2: Axial image from CT scan of the chest shows fluid and inflammatory changes adjacent to the aortic arch (curved arrow), gas bubbles along the aortic wall (straight arrow) and left pleural effusion $(*)$. 


\section{Cureus}

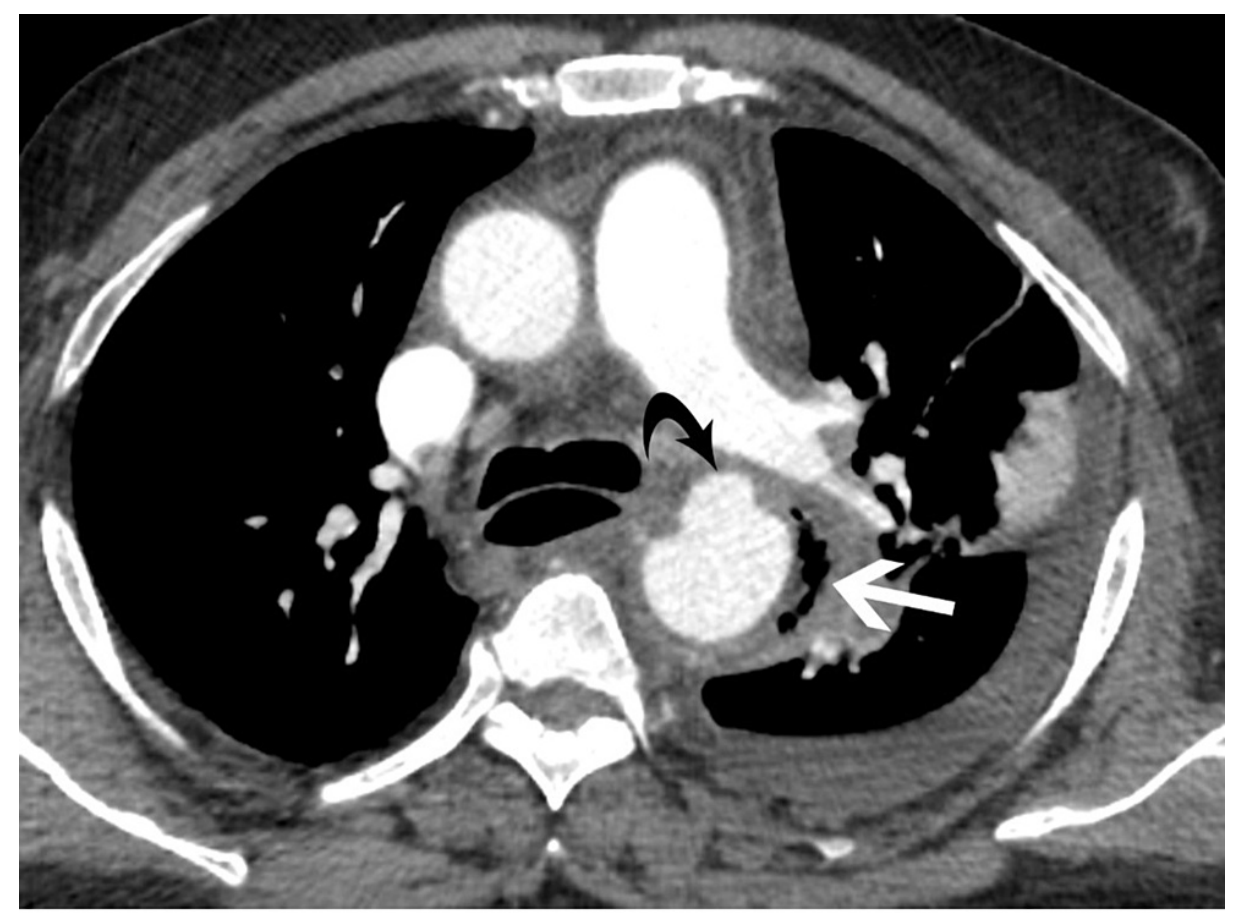

FIGURE 3: Axial image at a slightly lower level shows an aneurysm (curved black arrow) projecting from the anterior wall of the proximal descending aorta with gas bubbles (white arrow) along the left lateral wall. 


\section{Cureus}

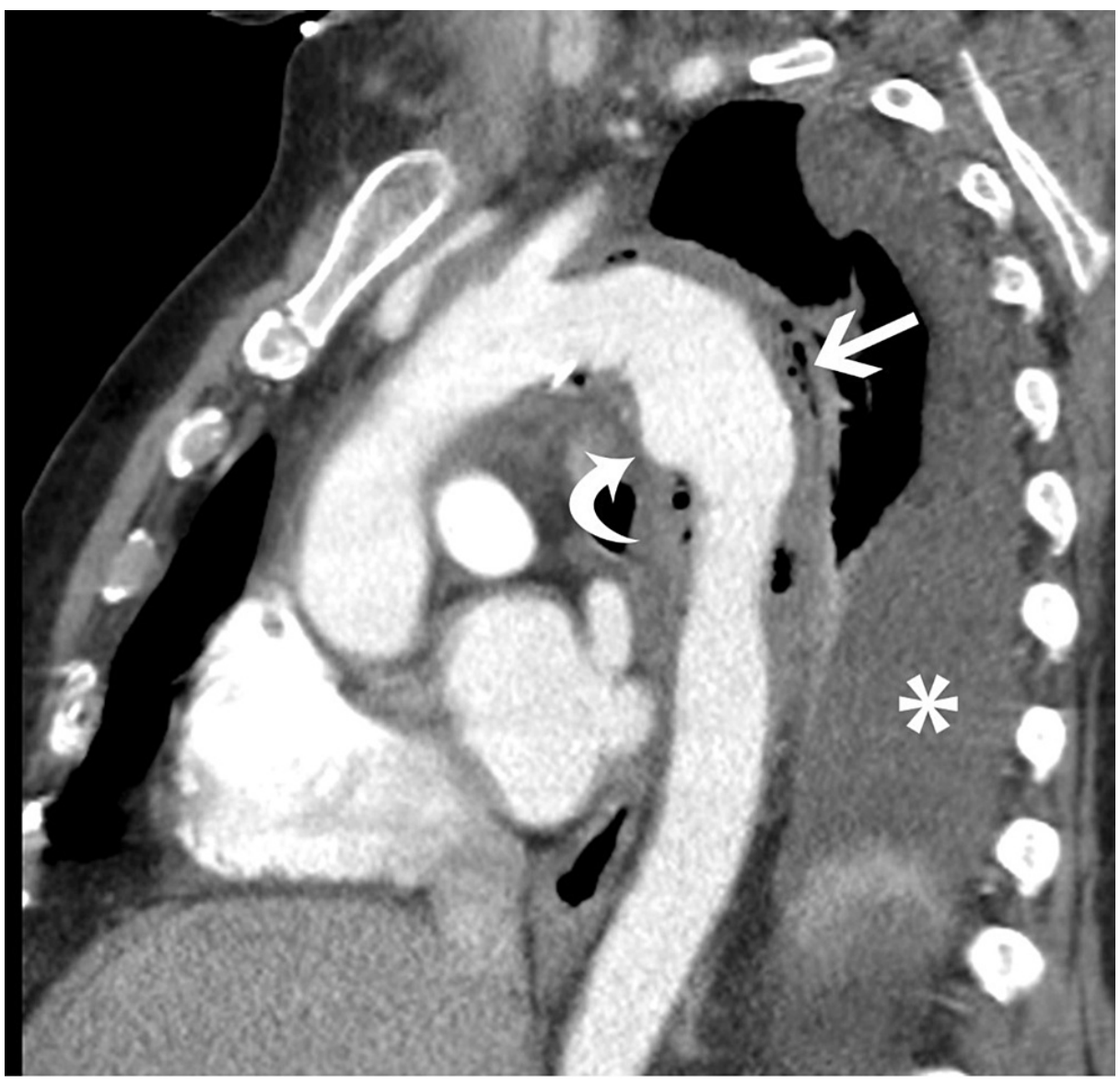

FIGURE 4: Oblique sagittal reformatted image in the plane of the aortic arch shows fluid and inflammatory changes along with gas bubbles (straight white arrow) along the aortic wall and aneurysm (curved white arrow) projecting anteriorly from the proximal descending thoracic aorta. Note left pleural effusion (*).

Blood cultures revealed Clostridium subterminale bacteremia. The patient was worked up to determine the primary source of bacterial infection. Since Clostridial infection can be associated with pathology in the gastrointestinal tract, the patient underwent colonoscopy and esophagogastroduodenoscopy, which were both negative. Abdominal CT also showed no evidence of gastrointestinal lesion or malignancy. Echocardiography, performed to exclude seeding from endocarditis, was also negative. The patient urgently underwent ascending aorta and distal arch reconstruction with a left carotid-subclavian bypass. Unfortunately, despite multiple revisions of the aortic graft, broad-spectrum antibiotics, and vasopressor support, the patient succumbed to cardiac arrest from multi-organ failure due to septic shock. Post-mortem aortic tissue cultures were positive for $C$. innocuum.

\section{Discussion}

Emphysematous aortitis is a rare but often fatal sequelae of mycotic aneurysm. Infection of the aorta can occur as a complication of surgery or interventional procedure, bacteremic seeding from a source in the gastrointestinal tract or endocarditis, or spread of infection from a source contiguous to the aorta, such as lung or vertebra. Risk factors include intravenous drug use, history of recent vascular procedure, bacteremia, immunosuppression, atherosclerosis, and pre-existing aneurysms. Most cases are usually caused by Staphylococcus or Salmonella species, reported in $71 \%$ and $24 \%$ of cases, respectively. Clostridium species comprise approximately $0.7 \%$ of cases.

As Clostridia are gas-producing organisms, emphysematous changes are present in $92.6 \%$ of Clostridial mycotic aneurysms. Spore-forming obligate anaerobes, Clostridia can proliferate in necrotic or anoxic areas of injury such as the damaged endothelial tissue of vessels. The release of Clostridial toxins and enzymes, such as hyaluronidase and hemolysin, produces gas gangrene infection. Curiously, the patient was found to have bacteremia with two different Clostridium species, C. subterminale on blood cultures and C. innocuum in the aortic wall. Clostridium bacteria are readily found in soil and in the gastrointestinal flora, and reported 
cases of $C$. subterminale bacteremia have been associated with gastrointestinal mucosal damage and colorectal malignancy. In rare cases, diverticulitis and ileus have been implicated as causes for Clostridium emphysematous aortitis. The presence of $C$. innoccum infection is particularly notable, as most Clostridium infections in the aortic wall are caused by C. perfringens or C. septicum [2]. Clostridium innoccuum has been associated with severe acquired immunodeficiency syndrome (AIDS), leukemia, or underlying malignancy, none of which our patient had. Our patient's negative gastrointestinal workup suggests a different source of infection for both species. Diabetes mellitus has been associated with gas gangrene infections in patients without preceding trauma and was likely a risk factor for our patient. We speculate that contamination of the patient's arteriovenous fistula may have resulted in bacteremic seeding of the bloodstream of the aorta during the hemodialysis.

The diagnosis of emphysematous aortitis involves a combination of findings. The typical presentation of this disease is characterized by pain, fever, and signs of infection, along with history of relevant risk factors including arterial disease or an immunocompromised state [3]. Of those diagnosed, $77.3 \%$ of patients presented with pain, most commonly abdominal and back pain, $45.5 \%$ presented with fevers, and $9.1 \%$ had gastrointestinal symptoms such as nausea, vomiting, and diarrhea, as in our case [4]. Laboratory findings that support the diagnosis include elevated inflammatory markers, presence of bacteremia, and positive aneurysm tissue cultures from the affected blood vessel.

Findings on CT imaging suggestive of an infected thoracic aneurysm include the presence of a saccular or multilobed aneurysm with inflammatory changes in the mediastinal fat adjacent to the aorta and perivascular fluid. Air along the aortic wall is diagnostic of emphysematous aortitis from gas-forming bacterial infection. When recognized, this requires urgent and aggressive management.

Treatment of mycotic aneurysms involves prompt resection and debridement of infected tissues and longterm antibiotic therapy. Patients with mycotic aneurysms also require antibiotic coverage for Staphylococcus and Salmonella infections. Duration of treatment can last up to six weeks or more depending on the pathogenic organism, patient comorbidities, and severity of disease. However, despite aggressive treatment, many patients suffer poor outcomes. The overall hospital mortality of patients with mycotic aortic aneurysms was $23 \%-31 \%$ with $7.7 \%$ mortality within the first 30 days after surgery. Causes of death include aneurysm rupture, progression of mediastinitis, graft infection, pneumonia, or multi-organ failure from septic shock [5].

\section{Conclusions}

Emphysematous aortitis is a rare consequence of mycotic aneurysms and is associated with high morbidity and mortality. Diagnosis can be presumed when imaging and laboratory findings are consistent with acute infection and confirmed by bacterial culture. Treatment involves both aggressive surgical reconstruction of the damaged vessel as well as long-term intravenous antibiotic therapy. The presence of air in the aortic wall with surrounding inflammatory changes on CT should prompt urgent and aggressive management.

\section{Additional Information \\ Disclosures}

Human subjects: Consent was obtained or waived by all participants in this study. Conflicts of interest: In compliance with the ICMJE uniform disclosure form, all authors declare the following: Payment/services info: All authors have declared that no financial support was received from any organization for the submitted work. Financial relationships: All authors have declared that they have no financial relationships at present or within the previous three years with any organizations that might have an interest in the submitted work. Other relationships: All authors have declared that there are no other relationships or activities that could appear to have influenced the submitted work.

\section{References}

1. Brook I: Anaerobic bacteria as a cause of mycotic aneurysm of the aorta: microbiology and antimicrobial therapy. Curr Cardiol Rev. 2009, 5:36-9. 10.2174/157340309787048095

2. Crum-Cianflone N: Clostridium innocuum bacteremia in a patient with acquired immunodeficiency syndrome. Am J Med Sci. 2009, 337:480-2. 10.1097/MAJ.0b013e31819f1e95

3. Sörelius K, Budtz-Lilly J, Mani K, Wanhainen A: Systematic review of the management of mycotic aortic aneurysms. Eur J Vasc Endovasc Surg. 2019, 58:426-35. 10.1016/j.ejvs.2019.05.004

4. Dubois M, Daenens K, Houthoofd S, Peetermans WE, Fourneau I: Treatment of mycotic aneurysms with involvement of the abdominal aorta: single-centre experience in 44 consecutive cases. Eur J Vasc Endovasc Surg. 2010, 40:450-6. 10.1016/j.ejvs.2010.07.017

5. Aoki C, Fukuda W, Kondo N, Minakawa M, Taniguchi S, Daitoku K, Fukuda I: Surgical management of mycotic aortic aneurysms. Ann Vasc Dis. 2017, 10:29-35. 10.3400/avd.oa.16-00117 